\title{
Spectrum of Paediatric Intestinal Obstruction in a Tertiary Hospital in Enugu, Nigeria
}

\author{
Chukwubuike Kevin Emeka ${ }^{1,}$, Ozor Ignatius Ikemefuna ${ }^{1}$, Enebe Joseph Tochukwu ${ }^{2}$ \\ ${ }^{1}$ Department of Surgery, Enugu State University Teaching Hospital, Enugu, Nigeria \\ ${ }^{2}$ Department of Obstetrics and Gynecology, Enugu State University Teaching Hospital, Enugu, Nigeria
}

Email address:

chukwubuikeonline@yahoo.com (C. K. Emeka)

${ }^{*}$ Corresponding author

\section{To cite this article:}

Chukwubuike Kevin Emeka, Ozor Ignatius Ikemefuna, Enebe Joseph Tochukwu. Spectrum of Paediatric Intestinal Obstruction in a Tertiary Hospital in Enugu, Nigeria. European Journal of Preventive Medicine. Vol. 7, No. 6, 2019, pp. 112-116. doi: 10.11648/j.ejpm.20190706.14

Received: October 16, 2019; Accepted: November 7, 2019; Published: November 17, 2019

\begin{abstract}
Background: Paediatric intestinal obstruction is a common surgical emergency and is associated with lots of morbidity and mortality especially in developing countries. Paediatric intestinal obstruction differs from adult intestinal obstruction in terms of aetiology, presentation, management and outcome. This study reports the demography, diagnosis, management and outcome of paediatric intestinal obstruction in pediatric surgical unit of a tertiary hospital in Enugu, Nigeria. Methodology: This was a retrospective study of paediatric patients who presented with intestinal obstruction to the paediatric surgical unit of Enugu State University Teaching Hospital (ESUTH) Enugu, South East Nigeria. Children above one month of age but below 15 years, who presented with symptoms and signs of intestinal obstruction, were recruited for the study. Intestinal obstruction in neonates was excluded from this study. The study period was for one year, from January $1^{\text {st }} 2018$ to December $31^{\text {st }} 2018$. Result: During the period of the study 45 cases, $30(66.7 \%)$ males and $15(33.3 \%)$ females were managed. The mean age of the patients was 52.2 months. Abdominal pain was the most common presenting symptom and intussusception was the most common cause of intestinal obstruction. Forty two patients $(93.3 \%)$ did well and were discharged. Two patients (4.4\%) died while one patient $(2.2 \%)$ was discharged against medical advice. Conclusion: Intussusception was the most common cause of intestinal obstruction in the current study. The mortality of $4.4 \%$ recorded in this study can be improved upon.
\end{abstract}

Keywords: Intestinal Obstruction, Paediatrics, Intussusception

\section{Introduction}

Intestinal obstruction occurs when the normal flow of intestinal contents is interrupted. This obstruction can occur at any level of the intestinal tract. Intestinal obstruction is a surgical emergency because of potential for intestinal ischaemia leading to perforation and peritonitis [1]. It is a common indication for emergency surgical procedure and can occur at any age. Paediatric intestinal obstruction differs from adult intestinal obstruction in terms of aetiology, presentation, management and outcome [2,3]. Paediatric intestinal obstruction is one of the most common causes of acute abdomen and is a common clinical condition a paediatric surgeon is called to review. Paediatric intestinal obstruction accounts for about $42 \%$ of all abdominal emergencies and $1 \%$ of all paediatric admissions [4]. The aetiology of intestinal obstruction can be congenital or acquired, mechanical or non-mechanical, extrinsic or intrinsic. The aetiology and pattern of paediatric intestinal obstruction varies based on geographical region and age distribution [5]. Children that have intestinal obstruction present with one or more of the following: abdominal pain, vomiting, constipation and/or abdominal distension. The symptomatology depends on the part of the intestine that is obstructed. For example, patient that has duodenal obstruction presents with vomiting and minimal or no abdominal distension, while patient with Hirschsprung's disease presents with marked abdominal distension due to chronic constipation. In developing countries, the outcome of paediatric intestinal obstruction is still far from encouraging with significant morbidity and mortality. This is due to late 
presentation, lack of paediatric intensive care unit and limited resources. However in developed countries, improvements in diagnostic investigations, proper surgical/anaesthetic techniques and good perioperative care have brought improved outcome [6].

The purpose of this study was to determine the aetiology, clinical presentation, management and outcome of intestinal obstruction in children in a tertiary care hospital in Enugu, South East Nigeria.

\section{Methodology}

This was a retrospective study of paediatric patients who presented with intestinal obstruction to the paediatric surgical unit of Enugu State University Teaching Hospital (ESUTH) Enugu, South East Nigeria. Paediatric patients older than 1 month but less than 16 years (one month to fifteen years) were recruited into the study. Intestinal obstruction in neonates was excluded from this study. The study period was for one year, from January $1^{\text {st }} 2018$ to December $31^{\text {st }} 2018$.

Enugu State University Teaching Hospital is a tertiary hospital located in Enugu, South East Nigeria. Enugu State University Teaching Hospital serves the whole of Enugu State, which according to the 2016 estimates of the National Population Commission and Nigerian National Bureau of Statistics, has a population of about 4 million people and a population density of $616.0 / \mathrm{km}^{2}$. The hospital also receives referrals from its neighbouring states. Diagnosis of intestinal obstruction was made based on the patients' clinical presentation and examination findings by the pediatric surgeon. Necessary investigations such as abdominal ultrasound and x-rays were done to support or disprove the diagnosis. Data were extracted from the case notes, operation notes, operation register, and admission-discharge records. The information extracted include the age, gender, presenting symptoms, diagnosis, duration of symptoms before presentation, time interval between presentation and intervention, treatment offered, complications of treatment, outcome and duration of hospital stay. Ethical approval was obtained from the Ethics and Research committee of Enugu State University Teaching Hospital. Statistical Package for Social Science (SPSS) version 23 was used for data entry and analysis. Data were expressed as percentages and mean.

\section{Result}

\subsection{Patients' Demography}

There were 50 cases of paediatric intestinal obstruction seen during the study period but only 45 cases had complete case records and formed the basis of this report. A total of 5,625 children were admitted into the children's ward of the hospital during the study period, out of which intestinal obstruction accounted for $0.8 \%$. There were $30(66.7 \%)$ males and $15(33.3 \%)$ females, with a male to female ratio of $2: 1$. The mean age of the patients was 52.2 months (range: 1 180 ) and the mean length of hospital stay was 12.6 days (range: 3-24). The mean duration of symptoms before presentation was 2.4 days (range: $1-3$ ). Six patients (13.3\%) presented to the hospital within 24 hours of onset of their symptoms, eleven patients $(24.4 \%)$ presented between 24 and 72 hours and twenty eight $(62.2 \%)$ presented after 72 hours. The mean duration between presentation and operative intervention was 2.0 days (range: 1-3). Eight patients $(17.8 \%)$ had operative treatment within 24 hours of presentation to the hospital; twenty six patients $(57.8 \%)$ had surgery 24 to 72 hours after presentation while eleven patients $(24.4 \%)$ had surgery 72 hours after presentation to the hospital, as shown in Table 1.

Table 1. Demographic characteristics of the patients.

\begin{tabular}{ll}
\hline Gender & $30(66.7 \%)$ \\
Male & $15(33.3 \%)$ \\
Female & 52.2 months (range: $1-180)$ \\
Mean age & 12.6 days (range: $3-24)$ \\
Mean length of hospital stay & 2.4 days (range: $1-3)$ \\
Duration of symptoms before & $6(13.3 \%)$ \\
presentation (mean) & $11(24.5 \%)$ \\
Less than 24 hours & $28(62.2 \%)$ \\
24 to 72 hours & 2.0 days (range: $1-3)$ \\
More than 72 hours & $8(17.8 \%)$ \\
Duration from presentation and & $26(57.8 \%)$ \\
surgery (mean) & $11(24.4 \%)$ \\
Less than 24 hours & \\
24 to 72 hours & \\
More than 72 hours &
\end{tabular}

\subsection{Clinical Features}

The patients presented with a wide range of symptoms which included abdominal pain, vomiting, abdominal distension and constipation. Most of the patients (53.2\%) presented with abdominal pain. Some of the patients presented with more than one symptom. The frequencies of occurrence of the symptoms are shown in Table 2.

Table 2. Clinical presentations of the patients.

\begin{tabular}{lll}
\hline Symptom & Number & Percentage (\%) \\
\hline Abdominal pain only & 24 & 53.2 \\
Abdominal pain plus distension & 7 & 15.6 \\
Abdominal distension only & 7 & 15.6 \\
Abdominal pain plus vomiting & 3 & 6.7 \\
Constipation & 3 & 6.7 \\
Vomiting & 1 & 2.2 \\
\hline
\end{tabular}

\subsection{Investigations}

Investigations done in our patients included abdominal ultrasound, abdominal $\mathrm{x}$ ray and computed tomography (CT) scan. Thirty six patients $(80 \%)$ did abdominal ultrasound, eight patients $(17.8 \%)$ did abdominal $\mathrm{x}$ ray while one patient (2.2\%) had computed tomography scan (Table 3 ).

Table 3. Investigations done by the patients.

\begin{tabular}{lll}
\hline Investigation done & Number & Percentage (\%) \\
\hline Abdominal ultrasound & 36 & 80 \\
Abdominal X ray & 8 & 17.8 \\
Computed tomography scan & 1 & 2.2 \\
\hline
\end{tabular}




\subsection{Diagnosis}

Intussusception was the most common pathology in our patients who had intestinal obstruction accounting for 20 cases (44.4\%). Typhoid intestinal perforation was the second most common which occurred in 8 cases $(17.8 \%)$. Seven patients $(15.6 \%)$ had obstructed external hernia which included inguinal and umbilical hernias. Five patients $(11.1 \%)$ had ruptured appendix while three patients $(6.7 \%)$ had Hirschsprung's disease. Two patients (4.4\%) were diagnosed with intestinal malrotation (Table 4).

Table 4. Clinical Diagnosis of the patients.

\begin{tabular}{lll}
\hline Diagnosis & Number & Percentage (\%) \\
\hline Intussusception & 20 & 44.4 \\
Typhoid intestinal perforation & 8 & 17.8 \\
Obstructed external hernia & 7 & 15.6 \\
Ruptured appendix & 5 & 11.1 \\
Hirschsprung's disease & 3 & 6.7 \\
Intestinal Malrotation & 2 & 4.4 \\
\hline
\end{tabular}

\subsection{Procedures Performed}

Out of the 20 children that had intussusception, ten of them $(22.2 \%)$ had right hemicolectomy with ileo-transverse anastomosis due to gangrenous bowel. Six (13.3\%) had hydrostatic reduction and four $(8.9 \%)$ were treated by manual reduction of intussusception. Nine patients (20\%) had laparotomy with intestinal repair. This included the 8 patients

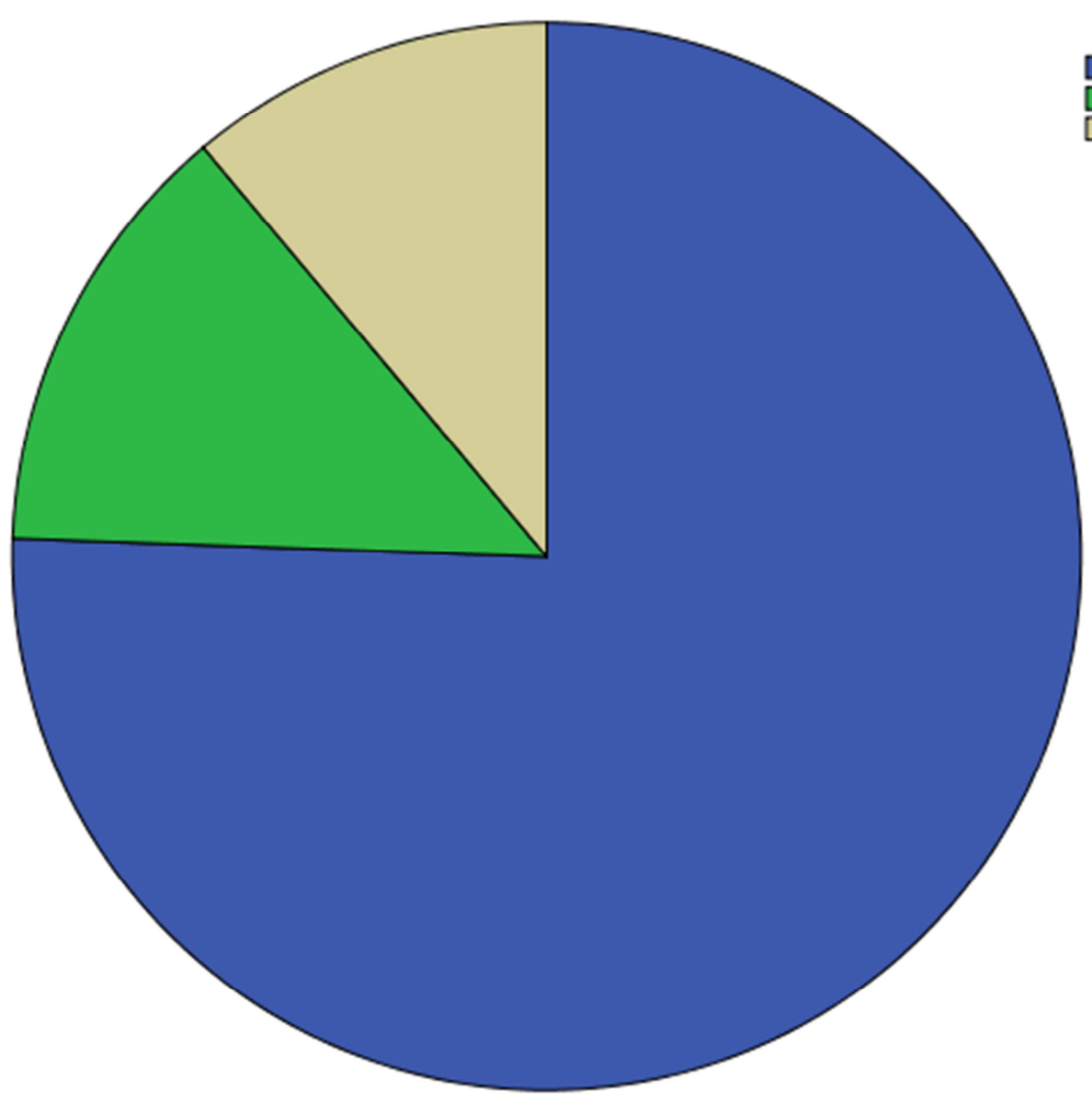

Figure 1. Pie Chart showing post-operative complications.
$(17.8 \%)$ that had typhoid intestinal perforation and one patient $(2.2 \%)$ that had obstructed inguinal hernia whose bowel was found to be gangrenous. The remaining 6 patients $(13.3 \%)$ that had obstructed hernias were treated by herniorrhaphy. Appendectomy and drainage of intra peritoneal abscess was done in five patients (11.1\%) that had ruptured appendix. Pull through (Swenson's) operation was the treatment carried out on the 3 children $(6.7 \%)$ that had Hirschsprung's disease. The 2 children (4.4\%) that had intestinal malrotation were treated by laparotomy and release of congenital bands causing bowel obstruction (Table 5).

Table 5. Procedures done on the patients.

\begin{tabular}{lll}
\hline Procedure & Number & Percentage (\%) \\
\hline Laparotomy plus right hemicolectomy & 10 & 22.2 \\
Hydrostatic reduction of intussusception & 6 & 13.3 \\
Laparotomy plus manual reduction & 4 & 8.9 \\
Laparotomy plus intestinal repair & 9 & 20 \\
Herniorrhaphy & 6 & 13.3 \\
Appendectomy plus abscess drainage & 5 & 11.1 \\
Pull through operation & 3 & 6.7 \\
Laparotomy plus adhesion release & 2 & 4.4 \\
\hline
\end{tabular}

\subsection{Post-operative Complications}

Thirty four patients $(75.6 \%)$ did not develop any complications. Six patients $(13.3 \%)$ developed surgical site infection while five patients (11.1\%) had severe sepsis (Figure 1).

\section{Complications \\ None \\ surgical site infection \\ severe sepsis}




\subsection{Outcome}

Forty two patients $(93.3 \%)$ did well and were discharged. Two patients $(4.4 \%)$ died while one patient $(2.2 \%)$ was discharged against medical advice.

\section{Discussion}

In paediatric surgical practice, intestinal obstruction is one of the most common emergencies encountered almost on daily basis and contributes significantly to the paediatric surgical disease $[5,7]$. Unlike in adults where post-operative adhesions and neoplasms account for most intestinal obstruction; intussusception and obstructed external hernias dominate intestinal obstruction in children $[8,9]$. Intestinal obstruction in children can be divided into neonatal and nonneonatal types [10]. Our focus on the current study was on non-neonatal intestinal obstruction.

The finding of male predominance in the current study is consistent with the findings of other series on paediatric intestinal obstruction $[4-6,8,11]$. The mean age of our patients is similar to the finding of Ahmed et al [12]. However, other researchers recorded wide variations in the mean age of their patients $[3,6]$. These differences may be explained by geographical variations. The duration of hospital stay of our patients is in line with the reports of some authors $[5,13]$, but varies with the reports of other authors [6, 9]. Differences in aetiological patterns of intestinal obstruction of the different studies may explain the findings of these reports. Delayed presentation of our patients, 24 to 72 hours after onset of their symptoms, is in agreement with the reports of other studies in sub Saharan Africa [3, 13, 14]. In developing countries, poverty and ignorance militate against early presentation to the hospital unlike what is obtainable in developed world [6]. With respect to the proportion of paediatric admissions that have intestinal obstruction, our finding of $0.8 \%$ is similar to that of Shah et al [5].

The most common symptom our patients presented with was abdominal pain. This is supported by the report of previous workers $[11,15,16]$. However, Ooko et al, Yang et al and Ogundoyin et al reported vomiting while Memon et al and Soomro et al reported constipation as the most common symptom in their series [3, 17-20]. Abdominal distension was observed by Pathak et al as the most common presenting symptom [21]. The explanation for these findings may be due to the predominant pathologies involved in the intestinal obstruction.

Imaging investigations is an integral part of management of intestinal obstruction. Huci in his report reiterated the importance of abdominal radiology, ultrasound and computed tomography scans in the evaluation of patients that have intestinal obstruction [1]. Plain abdominal radiograph is often regarded as the initial diagnostic imaging tool for patients presenting with abdominal symptom [22]. A study on paediatric intestinal obstruction done in Kenya recorded that
$72.8 \%$ of the patients had plain abdominal x ray [3]. Most of our patients $(80 \%)$ had abdominal ultrasound because the $\mathrm{x}$ ray machine had some issues during the period of the study. Again, ultrasound is easily accessible, non-invasive, radiation free and cheap imaging modality [23].

Intussusception remains the most common aetiology of intestinal obstruction in children as reported by many authors $[6,8,10,16,20,21]$. This is consistent with the finding of the current study. However, this finding is unsupported by the report of other workers $[2,3,5,13]$. At separate times and separate places, different authors have reported anorectal malformation, Hirschsprung's disease, Ascariasis and irreducible hernias as the most common causes of paediatric intestinal obstruction. Pattern of intestinal obstruction varies from one place to another and depends on the study population [3].

Most of our patients had intestinal resection and anastomosis. This is in agreement with the reports of other authors $[13,16,17,20]$. Only few of our patients $(13.3 \%)$ had non operative treatment (hydrostatic reduction of intussusception). These are patients who had intussusception and presented early to the hospital. However, Ooko et al and Mishra et al reported high number of their patients treated non-operatively $[3,11]$. The disease process involved and the time of presentation to the hospital may explain this difference in treatments given to the patients.

Surgical site infection was the most common complication in our patients. This finding is consistently observed in many other reports too $[6,8,11,16,20]$. The mortality rate in the current study is in agreement with that of other studies of its nature $[3,19]$. Documented mortality rates of children who have intestinal obstruction vary widely, from $0.9 \%$ to $20 \%$ [ 9 , 11]. Reduction in mortality rate may be explained by increased awareness, early presentation, prompt diagnosis, adequate treatment, improved anaesthetic/surgical techniques, potent antibiotics and better peri-operative care [21].

\section{Conclusion}

In the present study, 45 cases of intestinal obstruction of paediatric age group (excluding neonates) were studied during a period of one year (January 2018 to December 2018). Paediatric intestinal obstruction accounted for $0.8 \%$ of all paediatric admission. The most common cause of intestinal obstruction was intussusception (44.4\%), followed by typhoid intestinal perforation $(17.8 \%)$, obstructed external hernia $(15.6 \%)$, ruptured appendix (11.1\%), Hirschsprung disease $(6.7 \%)$ and intestinal malrotation (4.4\%). Abdominal pain only $(53.2 \%)$ was the most common symptom in this series, followed by abdominal pain plus abdominal distension (15.6\%), abdominal distension only (15.6\%), abdominal pain plus vomiting $(6.7 \%)$, constipation only $(6.7 \%)$ and vomiting only $(2.2 \%)$. Most of our patients $(62.2 \%)$ presented after 72 hours of onset of their symptoms. Abdominal ultrasound, laparotomy with right hemicolectomy and surgical site 
infection were the most common investigation, procedure and complication recorded in our patients respectively. Only $13.3 \%$ of the patients were managed non-operatively, the rest required operative intervention. The mortality rate in this study was $4.4 \%$.

\section{References}

[1] Huci T. Acute GI obstruction. Best Pract Res Clin Gastroenterol. 2013; 27 (5): 691-707. doi: 10. 1016/j.bpg.2013.09.001.

[2] Bhedi A, Prajapati M, Sarkar A. A prospective study of intestinal obstruction in paediatric age group. Int Surg J. 2017; 4 (6): 1979-1983. doi: 10.18203/2349-2902.isj20172395.

[3] Ooko PB. The spectrum of paediatric intestinal obstruction in Kenya. The Pan African Medical Journal. 2016; 24: 43. doi: 10.11604/pamj.2016.24.43.6256.

[4] Adejuyigbe O, Fashakin EO. Acute intestinal obstruction in Nigerian children. Trop Gastroenterol. 1989; 10 (1): 33-40.

[5] Mansi Shah, Jared Gallaher, Nelson Msiska, Sean E. McLean, Anthony $G$ Charles. Pediatric Intestinal Obstruction in Malawi: Characteristics and Outcome. Am J Surg. 2016; 211 (4): 722-726. doi: 10.1016/j.amjsurg.2015.11.024.

[6] Harissou Adamou, Ibrahim Amadou Magagi, Oumarou Habou, Ousseini Adakal, Kabirou Ganiou, Magagi Amadou. Acute mechanical intestinal obstruction in children at zinder national hospital, Niger: Aetiologies and prognosis. Afr J Paediatr Surg. 2017; 14 (3): 49-52.

[7] Ikeda H, Matsuyama S, Suzuki N, Takahashi A, Kuroiwa M, Hatakeyama S. Small boewl obstruction in children: review of 10 year experience. Acta Paediatr Jpn. 1993; 35 (6): 504-7.

[8] Houben CH, Pang KK, Mou WC, Chan KW, Tam YH, Lee KH. Epidemiology of small-bowel obstruction beyond the neonatal period. Annals of Pediatric Surgery. 2016; 12 (3): 9093. doi: 10.1097/01.XPS.0000481055.24776.db.

[9] Oladele AO, Akinkuolie AA, Agbakwuru EA. Pattern of intestinal obstruction in a semiurban Nigerian hospital. Niger J Clin Pract. 2008; 11 (4): 347-50.

[10] Gangopadhyay AN, Wardhan H. Intestinal obstruction in children in India. Pediatr Surg Int. 1989; 4: 84. doi: 10.1007/BF00181839.

[11] Mishra PK, Agrawal A, Joshi M, Sanghvi B, Shah H, Parelkar SV. Intestinal obstruction in children due to Azcariasis: A tertiary health centre experience. 2008; 5 (2): 65-70.

[12] Ahmed H. Al-Salem, Mohammad Oquaish. Adhesive
Intestinal Obstruction in Infants and Children: The Place of Conservative Treatment. ISRN Surgery. 2011; Article ID 645104, 4 pages. doi: 10.5402/2011/645104.

[13] Ntakiyiruta G, Mukarugwiro B. The Pattern of intestinal Obstruction at Kibogola Hospital, a rural Hospital in Rwanda. East and Central African Journal of Surgery. 2009; 14 (2): 103-108.

[14] Vanderkolk W, Snyder C, Figg D. Cecal-Colic Adult Intussusception as a Cause of Intestinal Obstruction in Central Africa. World J. of Surg. 1996; 20 (3): 341-344. doi: $10.1007 / \mathrm{s} 002689900055$.

[15] Akgun Y. Intestinal obstruction caused by Ascaris Lumbricoides. Dis Colon Rectum. 1996; 39: 1159. doi: 10.1007/BF02081419

[16] Gyedu A, Yifieyeh A, Nimako B, Amoah M, Abantanga FA. Intestinal obstruction in older children in Komfo Anokye Teaching Hospital. a tertiary referral centre in Kumasi, Ghana. Annals of Pediatric Surgery. 2015; 11 (1): 7-12. doi: 10.1097/01.XPS.0000455090.05043.54.

[17] Kwang-Ho Yang, Tae-Beom Lee, Si-Hak Lee, Soo-Hong Kim, Yong-Hoon Cho, Hae-Young Kim. Congenital adhesion band causing small bowel obstruction: What's the difference in various age groups, pediatric and adult patients? BMC Surgery. 2016; 16: 79. doi: 10.1186/s12893-016-0196-4.

[18] Ogundoyin OO, Afolabi AO, Ogunlana DI, Lawal TA, Yifieyeh AC. Pattern and outcome of childhood intestinal obstruction at a Tertiary hospital in Nigeria. Afr Health Sci. 2009; 9 (3): 170-3.

[19] Memon MA, Patel JL, Siddharth RK, Mahendra KD. A study on clinico etiological spectrum of intestinal obstruction in paediatric age group. International Journal of Research in Medical Sciences. 2016; 4 (8): doi: 10.18203/2320$6012 \mathrm{ijrms} 20162239$.

[20] Sirajuddin Soomro, Sikandar Ali Mughal. Intestinal Obstruction in Children. Journal of Surgery Pakistan (International). 2013; 18 (1): 20-23.

[21] Sushil Kumar Pathak, Sanjeev Kumar Sinha. Assessment of Intestinal Obstruction in Children. International Journal of Medical and Health Research. 2017; 3 (4): 160-162.

[22] Wendy Geng, Michael Fuller, Brooke Osborne, Kerry Thoirs. The value of the erect abdominal radiograph for the diagnosis of mechanical bowel obstruction and paralytic ileus in adults presenting with acute abdominal pain. J Med Radiat Sci. 2018; 65 (4): 259-266. doi: 10.1002/jmrs. 299.

[23] Magdalena Andrzejewska, Marian Grzymislawski. The role of intestinal ultrasound in diagnostics of bowel diseases. Prz Gastroenterol. 2018; 13 (1): 1-5. doi: 10.5114/pg.2018.74554. 